The International Journal of Multimedia \& Its Applications (IJMA) Vol.4, No.4, August 2012

\title{
Augmented Reality Platforms for Virtual FITTING ROOMS
}

\author{
Ioannis Pachoulakis and Kostas Kapetanakis \\ Department of Applied Informatics and Multimedia, Technological Educational Institute \\ of Crete, Heraklion-Crete, Greece \\ ipdepp.teicrete.gr \\ kapekost@epp.teicrete.gr
}

\begin{abstract}
Multi-sensor body scanners combined with new algorithms and social media technologies have started a revolutionary shift away from the classic desktop paradigm and into the direction of intuitive, "natural interaction" where people interface with the technological world through hand gestures, speech and body language. This article reviews recent examples of Virtual Fitting Rooms (VFRs) and supporting technologies which facilitate the shopping experience by letting customers to try-on apparel and/or mixand-match accessories without being physically present in the retail shop. These platforms are not only powerful decision tools for the on-line shopper, but also contribute to the fun factor of in-store shopping. Using depth scanning techniques, VFRs can create accurate 3D models of shoppers and meaningfully query retail digital catalogs, filter out non-fitting items and allow customers assess the styling and matching aspects in real time. In addition, omnipresent social networking features allow sending photos or videos of the shopper wearing the apparel for quick feedback. The quality of service provided by current VFRs is sufficiently high to boost sales but also minimize returns due to improper fit.
\end{abstract}

\section{KEYWORDS}

Augmented Reality, 3D scanning, cloth models, simulations

\section{INTRODUCTION}

Shopping is a time-consuming activity for some people, while for others a much enjoyed one. Many approaches have been tried in the not so distant past to make it possible to simultaneously answer two fundamental shopper concerns: "does it suit" and "does it fit", therefore reducing much of the guesswork involved in shopping. For example, by using 2D CAD patterns of actual garments that correspond to regular sizes commonly found on a shop floor, [1] present a methodology for producing 3D clothes with realistic behavior, while at the same time providing users with a "feel" for the garment's details. The 3D graphics technologies applied in [2] help create and simulate a virtual store. They also present a Web application which provides powerful access to and manipulation of garments to facilitate garment design, pattern derivation and sizing. The 3D geometrical modeling algorithm in [3] is based on the mapping of $2 \mathrm{D}$ objects on a 3D model and is incorporated in a methodology that can be applied to the automatic modeling of various "secondary" garment parts like collars, waist bands and pockets. The approach aims to automate the process of 3D garment modeling from flat patterns and leads to an intuitive interface for novice users.

Furthermore, [4] apply a pattern designers' draping technique which lays down cloth on a dummy and seeks a final pattern shape by iterative cutting. Real garment pattern design methods using chalks, pins and scissors are replaced with a NURBS cutting curve and mesh cutting algorithm. The process allows artificial cloth to be draped on a mannequin and cut and edited interactively. DOI : 10.5121/ijma.2012.4404 
The International Journal of Multimedia \& Its Applications (IJMA) Vol.4, No.4, August 2012

A method of applying geometry images to cloth simulation [5] purports to obtain cloth motion for surface meshes of arbitrary genus, while retaining the simplicity of a mass-spring model. That work also adapts an implicit/explicit integration scheme, utilizing the regular structure of geometry images, to improve performance. Additionally, the cloth is able to drape over other objects, also represented as geometry images. The method is efficient enough to allow real-time simulation of fairly dense cloth meshes. The study of [6] applies 3D technologies to assess garment fit in order to evaluate the usability of pattern customizing technologies. It presents primary data from experiments on the provision and testing of garment fit of specified size patterns for a jacket and skirt as well as findings on virtual and human fit trials and an evaluation of the 3D technology. The study evaluates procedures and presents problematic features of the 3D fitting software concluding that, although some issues concerning efficient provision and testing of fit still exist, 3D technology overall provides adequate evaluation of fit.

The simulation method in [7] is based on six pieces of Bezier curved surfaces and generates a 3D dress model by using 32 pieces Bezier curved surfaces. In addition, the method reaches satisfying simulation results quickly. The key aspects of Virtual Prototyping and enabling technologies in the fashion industry are discussed in [8] along with their benefits and shortcomings. In particular, available technologies in the conceptual and design phases of the fashion products development chain show that prototyping in a virtual environment is feasible for specific goals and applications. Indeed, methodologies that shorten the development and production time justify considerable investments. In order to provide online apparel stores with an easy way to build a 3D clothing show room, [9] proposes 3D garment generation using 2D garment photos instead of 2D CAD patterns. All essentials for duplicating a garment in 3D are extracted from a photo. A magnet-based interior nodes insertion method and Delaunay-based triangulation are used to produce uniform garment meshes. The back and front garment meshes are then automatically seamed around a virtual mannequin based on the mass-spring model. The aim of the research is to develop a convenient and friendly virtual show room for people who do not have access to $2 \mathrm{D}$ CAD patterns of real garments.

The design of camera-based technologies to support retail shopping in a physical store is explored in [10]. The method uses an implicitly controlled video technology that allows shoppers to directly compare currently worn garments with images from the previously worn garments, with the twist of orienting images from past trials to the shopper's current pose as he/she moves. The system also allows comparison to clothes that other people in the shoppers' social network are wearing. The clothing animation framework in [11] integrates several key clothing animation techniques. In addition, an interactive system is set up on the three-tier architecture that provides users with basic functionalities including cloth animation, human model editing and clothing simulation. The interactive editor provides a set of intuitive tools for animators to design a rich variety of human shapes and cloth animation. As a prototype system for clothing animation and virtual dressing, it is fast, reliable and expandable.

Driven by the human perception of garment sketches, [12] propose a context-aware garment sketch interpretation based on a set of observations about key factors that affect the shape of garments. Based on this analysis they develop a geometric method for sketch-based modeling of garments which obtains more realistic results than previous techniques. The effectiveness of this method is demonstrated on a variety of inputs while validation of the approach was performed via a user study where viewers were asked to compare the believability of our outputs versus previous ones. The fast 3D garment simulation method introduced in [13] generates 3D clothing models by using several pieces of Bezier curved surfaces based on body data. The work proposes the spatial analytic geometry algorithm by which determines the positions of control points. Furthermore, the cutting piece is simulated by fitting quadratic Bezier curves. Experiments show that the static models performed satisfactory with a rendering performance of 1-3 seconds, which is almost appropriate for online trying. 
The International Journal of Multimedia \& Its Applications (IJMA) Vol.4, No.4, August 2012

A system that allows users to interactively control their 3D model at home using a commodity depth camera is presented in [14]. The model can then be augmented with (downloaded) virtual clothes, resulting in private, enjoyable, virtual try-on experience. As a first step, the user needs to create his or her 3D using a multi-camera setup that captures him or her in a fraction of a second, after which a 3D model is created. The model is subsequently transmitted to the user's home system to serve as a realistic avatar for the virtual try-on application. The system provides freeviewpoint high-quality rendering with smooth animations and correct occlusion, and therefore improves the state of the art in terms of quality. It utilizes cheap hardware which makes it appealing to a wide audience.

The interactive garment design tool in [15] enables interactive bidirectional editing between 2D patterns and 3D high-fidelity simulated draped forms, providing for a continuous, interactive, and natural design modality in which $2 \mathrm{D}$ and 3D representations are simultaneously visible and seamlessly maintain correspondence. Artists can now interactively edit 2D pattern designs and immediately obtain stable accurate feedback online, thus enabling rapid prototyping and an intuitive understanding of complex drape form. Finally, [16] examines augmented reality solutions for e-commerce to show that it is possible to define a strong platform for marketing products and for advertising promotions. The approach is based on introducing computer vision in a hands-free augmented reality setting. The proposed framework is prepared to operate in two different environments: personal computers and public street marketing places.

Clearly then, Virtual Fitting Rooms (VFRs) have already started to revolutionize the state of affairs in both retail and e-tail industries by letting customers try-on apparel and mix-and-match accessories without being physically present in the retail shop. These applications draw on multiple technologies to finally make it possible address the "suit"/"fit" dilemma. On the hardware side, multi-sensor bars like Microsoft Kinect and ASUS Xtion have brought depth scanning to the masses by allowing accurate full-body depth maps and gesture recognition via supporting frameworks. In addition, VFRs rely heavily on Augmented Reality (AR), which employs specialized software and hardware to merge the digital and the physical worlds by immersing digital information into real video to generate persuasive looking scenes in real time.

Modern VFRs combine AR technologies with depth and color data to provide robust body recognition functionality and successfully address the fit and suit aspects of shopping. In addition, promising platforms offer real-time video simulations which allow customers to visualize products as part of their current outfits and view them from multiple angles. These platforms are not only powerful decision tools for on-line shopping but also increase the fun factor for in-store shopping. The present article reviews successful examples of VFRs and discusses fundamental technologies behind some of these shopping and advertising tools, many of which employ fullbody scans to offer only pieces of clothing with a satisfactory fit and at the same time facilitate the styling and matching aspects of shopping.

\section{NATURAL INTERACTION (NI) SENSORS AND SUPPORTING SOFTWARE PLATFORMS}

Natural Interaction (NI) is the ability to command and control the digital world using hands, body and voice, making traditional peripherals such as remote controls, keypads and mice obsolete. Based on technologies where the entire body is the controller, Natural User Interfaces (NUIs) deviate massively from the classic desktop paradigm, as they use body gesture recognition to navigate applications and complete tasks such as browsing, selecting, adding to shopping basket and checking out. At the same time, by accommodating gestures that by nature are highly personalized, NUIs are addressed to the entire population instead of only the technologically savvy. 
The International Journal of Multimedia \& Its Applications (IJMA) Vol.4, No.4, August 2012

Some of the basic players in the Virtual Fitting Room industry appear in Fig. 1, where current technological advances from multiple areas converge. Presently, the main NI players in the hardware sensor consumer market are Microsoft with Kinect and Asus with Xtion, both of which utilize the PS1080 chip from Prime-Sense in competing implementations. Real-time modeling of natural scenes with commodity sensors and graphics hardware has catapulted augmented reality (AR) to an entirely new level by allowing real-time reconstruction of dense surfaces with a level of detail and robustness beyond the capabilities of previously available solutions that used passive computer vision. These multi-sensor bar products collect synchronized audio, video and depth data streams to be manipulated to suit a rich gamut of applications using a number of software frameworks.

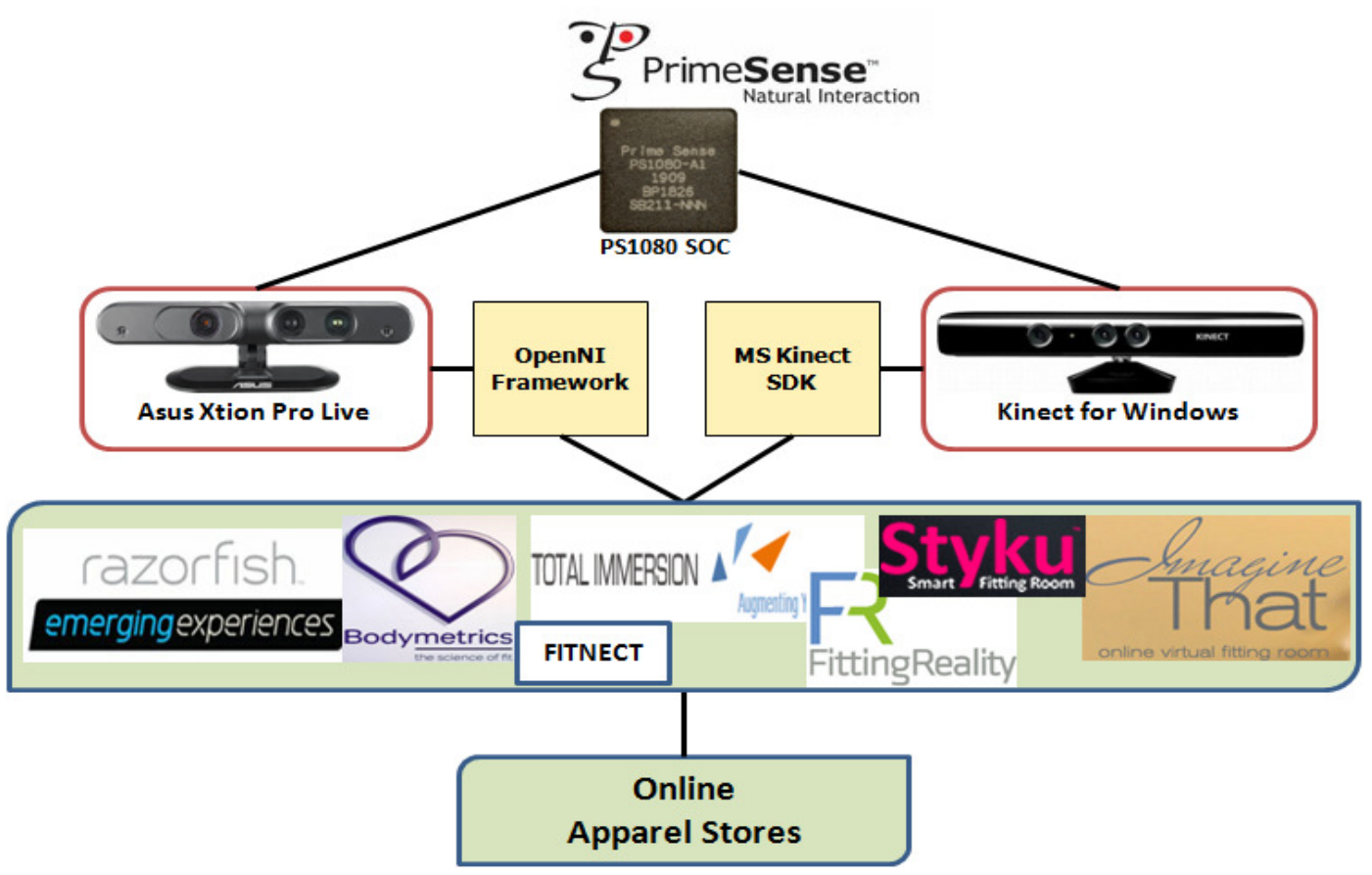

Figure 1. Various players in the Virtual Fitting Room Arena

Microsoft Kinect (an acronym which results from combining the words kinetic and connect) was marketed in November 2010 and is marketed in two versions: Kinect for the Xbox game console and Kinect for Windows, the latter of which sells at a higher price and incorporates enhancements such as "near mode" operation, skeletal tracking control, API improvements, improved USB support across a range of Windows computers and Windows-specific acoustic models [17]. As a voice-activated console that incorporates video capturing and facial recognition capabilities, Kinect can be employed in a wide range of applications. Microsoft has also designed Kinect as an enhancement to the Xbox Live experience. Xbox Live Video Chat makes use of Kinect's cameras and microphones for a webcam-like live chat with multiple friends at once.

The Xtion from Asus exists in three versions: Xtion, Xtion Pro and Xtion Pro Live. The last two of these are bundled with drivers, OPNI NITE middleware compatible SDK and are addressed to developers. The Live version basically adds an SXGA RGB camera for live image/video capture. Both Kinect and Xtion PRO LIVE use infrared sensors, adaptive depth detection technology, color image sensing and audio stream to capture a user's real-time image, movement, and voice, making user tracking more precise. The main characteristics of the Kinect and Xtion sensors appear in Table 1. 
The International Journal of Multimedia \& Its Applications (IJMA) Vol.4, No.4, August 2012

Table 1. MS Kinect and Asus Xtion (Pro-Live version) pertinent characteristics.

\begin{tabular}{|c|c|c|}
\hline Specification & Microsoft Kinect & Asus Xtion Pro-Live \\
\hline Field of View & $57^{\circ} \mathrm{H}, 43^{\circ} \mathrm{V}$ & $58^{\circ} \mathrm{H}, 45^{\circ} \mathrm{V}$ \\
\hline Sensors & RGB \& Depth & RGB \& Depth \\
\hline Depth Range & $1.2 \mathrm{~m}-3.5 \mathrm{~m}$ & $0.8 m-3.5 m$ \\
\hline Depth Steam & QVGA (320x240) 16-bit @ 30 fps & $\begin{array}{l}\text { VGA (640x480) @ } 30 \mathrm{fps} \\
\text { QVGA (320x240) @ } 60 \mathrm{fps}\end{array}$ \\
\hline RGB Stream & VGA (640x480)32-bit @30 fps & SXGA (1280*1024)@30fps \\
\hline Microphones & 3 (left) +1 (right) & 2 mikes for stereo capture \\
\hline Power Supply & $12 \mathrm{~V}$ DC $+5 \mathrm{~V}$ USB connection & 5V USB connection \\
\hline OS Support & Win7 32/64 & $\begin{array}{l}\text { Win 32/64: XP, Win } 7 \\
\text { Linux Ubuntu 10.10: X86,32/64 bit }\end{array}$ \\
\hline $\begin{array}{l}\text { Dimensions / } \\
\text { Weight }\end{array}$ & $12 " \times 3 " x 2.5 "$ at $3.0 \mathrm{lb}$ & $7 "$ x 2" x $1.5 "$ at $0.5 \mathrm{lb}$ \\
\hline
\end{tabular}

The supporting software environments for both sensors enable developers to create custom gesture-based applications without the need to write complex programming algorithms:

- Gesture detection: The ability to track hand motions in real-time, thus turning the user's hands into controllers.

- Full-body detection: The ability to track the users' full body movement with support for multiple player recognition, making it ideal for full-body gaming.

- RGB image sensing captures real-time video scenes that can be used separately or in combination to the synchronized depth-image data to various application-specific purposes.

- Audio streaming allows support for voice control and other voice recognition applications.

Mainstream development environments for NI sensors such as Kinect and Xtion include the OpenNI Framework, the Microsoft Kinect SDK and the Point Cloud Library. The Open Natural Interaction (OpenNI) [18] organization is an industry-led, non-profit organization formed to certify and promote the compatibility and interoperability of NI devices, applications and middleware. The organization includes partners like PrimeSense (a semiconductor company headquartered in Tel Aviv, Israel, with expertise in natural interaction and 3D depth sensing solutions) [19], Willow Garage (personal robotics applications) [20], Side-Kick (motion control games) [21], Asus (provides hardware such as the Xtion for OpenNI applications) [22] and AppSide (end-to-end content marketplace for motion-controlled entertainment devices) [23]. PrimeSense produces the PS1080 SOC, the heart of NI hardware such as MS Kinect and ASUS Xtion. The chip synchronizes the depth image, color image and audio streams obtained from the device's cameras and microphones and implements in hardware all depth acquisition algorithms. In addition, it provides a USB 2.0 interface to communicate data to the host.

OpenNI is a multi-language ( $\mathrm{C}, \mathrm{C}++, \mathrm{C \#}$, Java), cross-platform open-source, freely downloadable and documented [18] framework that defines APIs for writing applications utilizing Natural Interaction. OpenNI provides an Application Programming Interface (API) to allow communication with both low level devices (e.g. vision and audio sensors), as well as high-level middleware solutions (e.g. tracking using computer vision) and can be used to develop applications utilizing natural interaction. OpenNI APIs are composed of a set of interfaces for 
The International Journal of Multimedia \& Its Applications (IJMA) Vol.4, No.4, August 2012

writing NI applications. The main purpose of OpenNI is to form a standard API that enables communication with both (a) vision and audio sensors (i.e., the actual devices that "see" and "hear") and (b) vision and audio perception middleware (i.e., the software components that analyze and handle the streamed audio and visual data from the scene). For example, software that receives visual data, such as an image, returns the location of the palm of a hand detected within the image.

The OpenNI Framework provides the interface for physical devices and for middleware components. Its architecture is built around modules, production nodes and production chains.

- OpenNI modules are hardware- or middleware-specific program units enabled by the OpenNI Framework API that are registered in the OpenNI framework and subsequently used to produce sensory data.

- OpenNI Production Nodes are sets of units that have a productive role in the process of creating the data for NI-based applications. For example, a User Generator is a production node that produces body data and a Depth Generator is a production node that takes raw sensory data from a depth sensor and outputs a depth map.

- OpenNI Production Chains allow the simultaneous registration of several modules to a single OpenNI implementation. The process makes the selection of specific sensor devices and middleware components with which to produce and process data more flexible and at the same time creates more robust and reliant optional node sequences.

Finally, OpenNI Arena [24] is a forum where developers can get information on how to create and upload OpenNI-compliant applications.

The Kinect algorithm discussed in [25] uses a low-cost depth camera and commodity graphics hardware to create accurate real-time depth mappings of complex and arbitrary indoor scenes in variable lighting conditions. The described system works well for mapping volumes up to seven cubic meters. In addition, Kinect utilizes face and voice recognition to identify a given person in a group, a very useful feature for group games. Microsoft Kinect SDK v1.0 [26] provides access to the desired functionality in Windows 7 Visual Studio.NET environments. Version 1.5 of the SDK is expected by end of May 2012 [27] and promises "seated" or 10-joint skeletal tracking, providing the capability to track the head, neck and arms of either a seated or standing user in both default and near mode. A comparison of the MS Kinect and PrimeSense OpenNI SDKs exists at [28].

The Point Cloud Library [29] is a large scale, open-source project for 3D point cloud processing [30]. It is released under the terms of the Berkeley Software Distribution (BSD) license and is free for commercial and research use. The PCL framework contains numerous state-of-the art algorithms including filtering, feature estimation, surface reconstruction, registration, model fitting and segmentation. These algorithms can be used to filter outliers from noisy data, stitch 3D point clouds together, segment relevant parts of a scene, extract keypoints and compute descriptors to recognize objects in the world based on their geometric appearance, as well as create surfaces from point clouds and visualize them. The short videos in [31] briefly compare the capabilities of KinectFusion and PCL.

To simplify development, PCL is organized in a set of modular libraries which can be compiled separately. This modularity is important for distributing PCL on platforms with reduced computational or size constraints. Source code and prebuilt binaries for Linux, Windows and Apple Mac OS X are currently available for download [30]. The code relies heavily on the NVidia CUDA [32] development libraries for GPU optimizations and requires a compatible GPU 
The International Journal of Multimedia \& Its Applications (IJMA) Vol.4, No.4, August 2012

for best results. The system is continuously refined and improved by a world-wide group of dedicated developers to model larger scale environments in the near future. Finally, a stable release (version 2.0) is expected in 2012.

\section{VIRTUAL FITTING ROOM (VFR) APPLICATIONS}

VFR-enabled shopping is available for stores, homes and smart mobile devices. Successful VFR applications, successful representatives of which will be discussed presently, automatically generate precise body scan data to guarantee the quality of fit. These "Body Shape" IDs can be manipulated to various purposes. One is to create accurate 3D avatar-type models of the customer's body on which to fit clothes as an alternative to fitting on the shopper's image itself. Another way to go is to combine $3 \mathrm{D}$ scan data with information such as gender and age to meaningfully query virtually unlimited clothing databases across multiple retailers, to order for family and friends, etc. Furthermore, granting access to accurate 3D models of garments and apparel from designers enables VFR technologies to incorporate physics to enhance and make more realistic the shopping experience. In addition, the incorporation of the communication and feedback capabilities of social media to obtain advice and feedback (e.g., tweeter, facebook) make the shopping experience even more enjoyable. Finally, smart-phone applications may also alert users for wish-listed items available in nearby stores to so that local shopping and deal finding can be usefully combined.

By successfully addressing the suit/fit dilemma, VFRs present unique opportunities for retailers in multiple directions. Shopper assurance of a "perfect fit" removes an important obstacle for online shoppers and should result in increased sales and decreased return rates. Multiple marketing channels increase on-line brand visibility. Interest shown on a per-item or per-category basis can also be utilized on a per-individual or at an aggregate (e.g., regional) level. In the following we will review some of the most successful applications in the Virtual Fitting Room industry.

\subsection{KinectShop from Razorfish Emerging Experiences}

KinectShop is an on-line AR shopping platform created by the Emerging Experiences team at Razorfish. Based on the Xbox Kinect sensor, the platform translates customer's real-life motions into on-screen movements to allow browsing through an unlimited virtual shelf of accessories and select apparel to try on in true 3D in front of a big-screen TV that acts as a virtual mirror Fig. 2(a). In addition, customers can use the Kinect RGB camera to create an album of different looks at multiple angles to share with friends via social networks, collect votes (likes and dislikes) and also store their wish-list on their smart-phones for on-the-go shopping. Social networking can also be employed to alert customers for the availability of wish-listed items in nearby stores at discount prices.

\subsection{Bodymetrics}

In a partnership with PrimeSense, Bodymetrics [33], a London-based technology company, has created 3D body scanning solutions for both retail stores [34], [35] and home customers. The retail version of the body scanner uses eight PrimeSense 3D sensors to map the customer's body for quick and accurate calculation of 100 measurements which are subsequently used by "Fit Stylists" to find garments that best suit the customer's unique shape and size. The home buyer solution employs the same PrimeSense-based NI sensors like Microsoft Kinect and ASUS Xtion to allow shoppers to have their body data scanned in and saved to an on-line profile. 
The International Journal of Multimedia \& Its Applications (IJMA) Vol.4, No.4, August 2012

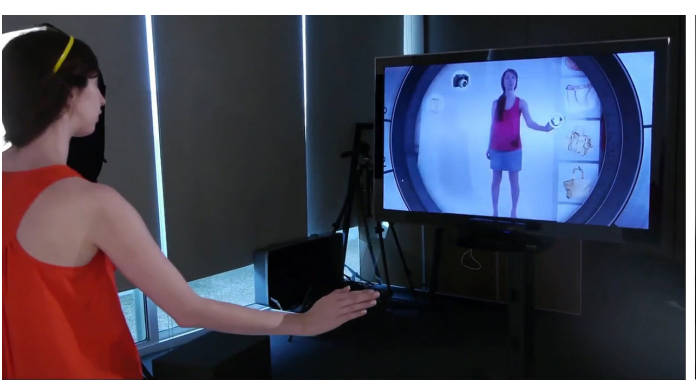

(a) KinectShop from Razorfish Emerging Experiences

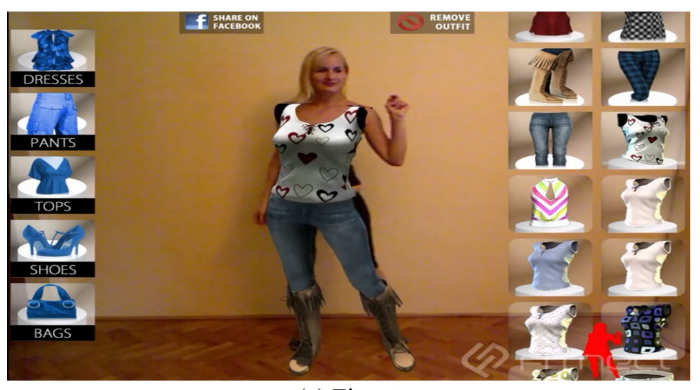

(c) Fitnect

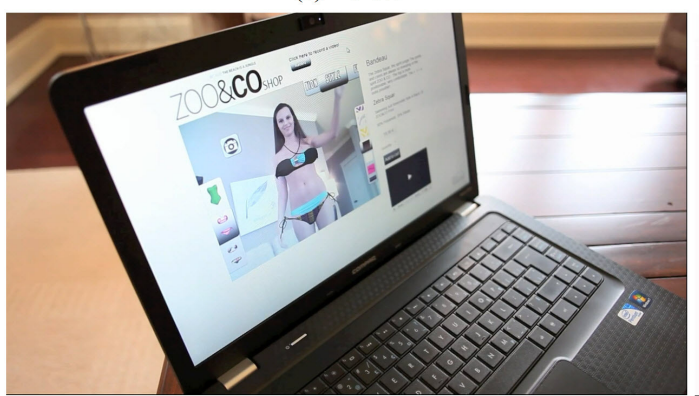

(e) Imagine That

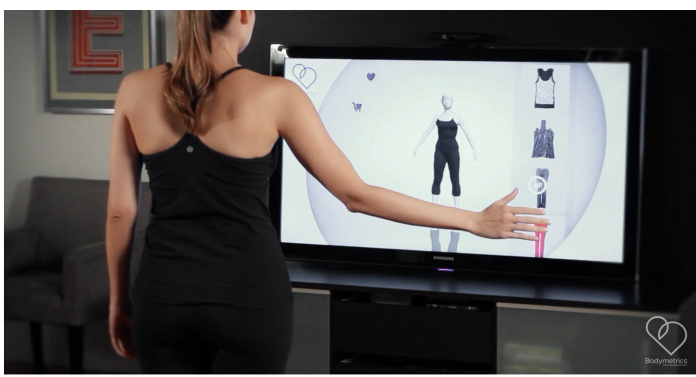

(b) Bodymetrics

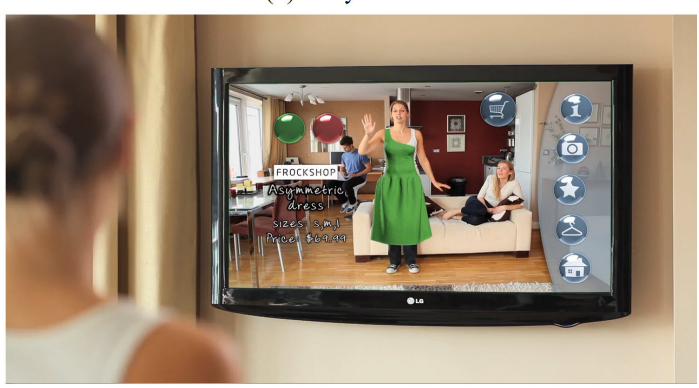

(d) Fitting Reality's VIPodium

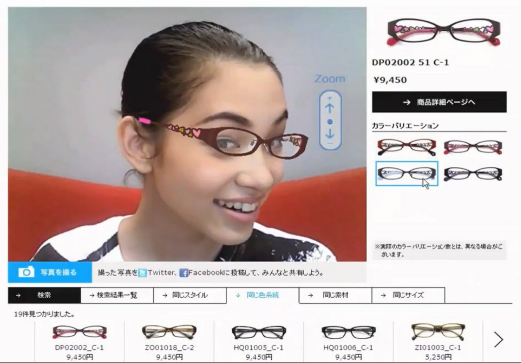

(f) Total Immersion's TryLive

Figure 1. Screen shots sampling mainstream functionality for reviewed Virtual Fitting Rooms

Bodymetrics partnered with the Razorfish Emerging Experiences team to incorporate this technology into an on-line shopping platform which uses the saved profile of a customer to (a) create an on-screen customer-sized avatar which follows the customer's motions through the NI sensor and (b) virtually try on various clothing types such as jeans, dresses, skirts and tops from connected partners, as seen in Fig. 2(b). In addition, as each piece of clothing is mapped onto the avatar's body, a color map is overlaid on the garment to depict the tight spots of the garment so that the customer can, for example, move on to another size or to an altogether different garment type. A retail version of the platform is deployed in stores and uses 3D scanning technology in a private booth to obtain an avatar that presumably corresponds even better to the customer than the home version which employs NI sensors like the MS Kinect and Asus Xtion. The "perfect fit" which is guaranteed from such technologies works to the benefit of both the customer (who can buy with increased confidence) and the retailer (who benefits from both increased sales and decreased rates of returns).

\subsection{Fitnect}

Fitnect implements an interactive AR virtual fitting room which uses the Kinect sensor as a 3D full-body capture device. A Natural Interaction user interface employs floating buttons to allow customers to browse the catalog and virtually try on garments of their choice (Fig. 2(c)). In addition, Fitnect uses 3D models of clothes and cloth physics for a more convincing experience. However, voids and empty spaces in the overlay imply that the solution is not as sophisticated as 
The International Journal of Multimedia \& Its Applications (IJMA) Vol.4, No.4, August 2012

that of Bodymetrics, suggesting that it can be used for styling and matching purposes but perhaps not for accurate sizing and fitting.

\subsection{VIPodium from Fitting Reality}

Moscow-based Fitting Reality [36] has created VIPodium, an AR fitting room application and shopping system which parses full body scanning image data to detect the physical outlines of a shopper and create a ShapeID, with which to query the product catalog of 3D virtual garments to offer pieces of clothing that perfectly fit that particular customer. A 3D interface allows intuitive navigation through the selected garments and item selection, after which a real-time video simulation of the customer "wearing" a 3D copy of a garment is created on screen so that the customer can assess the quality of fit and the style/matching aspects from multiple angles. VIPodium versions exist for home shoppers and for in-store usage (Fig. 2(d)). The former employs either Kinect for Windows connected to a computer/plasma TV or Kinect for Xbox hooked to an Xbox 360 console. For stores, VIPodium can be integrated in a shopping window to convert static retail fronts into interactive kiosks attracting attention to store brands, new collections and special offers. It can also function as a public apparel try-out place to enhance the shopping experience.

\section{5. "Imagine That" on-line Virtual Fitting Room}

The "Imagine That" [37] VFR moves along the lines of the previous solutions, providing 3D models of apparel, $360^{\circ}$ views of the customer, a natural interaction user interface based on hand gesture recognition and social media integration. The in-store kiosk version uses a big-screen TV as a full-body mirror on which it projects a live video stream of the customer from the NI sensor's RGB camera or a created avatar of the customer as well as menus for navigating through the onscreen inventory. The customer can select various items to mix-and-match, narrow the selection and tag favorites to show sales staff for an actual try-on. The on-line version claims full integration with existing web sites and uses body scanning and analytics technologies for virtual try-on in the comfort of the customer's own home (Fig. 2(e)). Social media integration also enhances the shopping experience.

\subsection{TryLive Eyewear from Total Immersion}

Total Immersion [38] provides AR commercial solutions such as the D'Fusion Studio Suite which enables the development of secured AR applications across a variety of fields and domains (digital marketing, event organizations and industrial applications). Total Immersion has released various commercial AR platforms for web, mobile and kiosk deployment under a common TryLive logo: Virtual Fitting Room, Eyewear, Watches, Shoes and Jewellery. TryLive Eyewear, in particular, requires a camera-equipped computer, tablet or smart phone and uses automated face recognition algorithms such as face shape classification to filter through a large database of fashion or optical eyewear. Customers can virtually try on selected items, preview them interactively from various angles, interchange lenses and frames for a customized fit, filter out selections and populate a shopping cart (Fig. 2(f)). In addition, pictures can be sent to friends via Facebook, Twitter or other social media for feedback.

\subsection{Apparel Manufacturing Made for You - AM4U}

Styku [39] virtual body-scanning partnered with Tukatech Apparel Technology, Critical Mass Manufacturing and AIMS apparel management system to develop a "smart fitting room" that directly connects customers to design and manufacturing. Their business model is based on "demand manufacturing" and goes a step further from other solutions by allowing for a garment to be cut, sewn, and shipped to the customer based on their exact body measurements and personal design preferences. 
The International Journal of Multimedia \& Its Applications (IJMA) Vol.4, No.4, August 2012

Styku's smart fitting room is based on the Microsoft Kinect sensor to scan the shopper's body and create an avatar-like virtual model matching the shopper's dimensions. Using Tukatech's [40] TUKAcad and TUKA3D software, customers may virtually try on clothes, ascertain the quality of the fit, identify areas that might be too loose or too tight and alter a pattern to fit their body's measurements. Using Styku's customization tool, consumers can design their own garment in 3D while viewing the quality of fit on their own custom avatar.

The resulting design, customized both for fit and appearance, is sent off to fabric printers like Critical Mass, where it is printed and dyed using innovative waterless and chemical-free technology. In the final step, the fabrics are then sewn into garments using traditional automatic sewing machines, pressed, and delivered to the customer.

Demand manufacturing works well with digital fabric printing solutions which do not require liquid dyes. The process enables dyeing, printing and imprinting only the required amount of fabric on a single machine, with a quick turnaround eliminating overestimated production runs and excess inventory. In addition to speeding up manufacturing and reducing excess production, companies also save time by not having to halt the manufacturing process to clean the dye press. The process works on a per-garment basis to produce every garment separately at manufacturing speed and, as such, requires no minimum orders. In addition, by producing only the amount of apparel that is sold, retailers can minimize financial loss due to excess production.

\section{CONCLUSIONS}

Internet retailing is a modern concept with a plethora of potential advantages and challenges associated with electronic retailing (a discussion appears in [41]). Providing consumers with correctly-fitting clothes is a top business concern in the garment industry. Virtual Fitting Rooms (VFRs) promise to revolutionize the state of affairs in both retail and e-tail industries by letting customers try-on apparel and mix-and-match accessories without being physically present in the retail shop. Recent sophisticated VFRs provide long-awaited answers to fundamental shopper concerns like "does it suit" and "does it fit" and reduce much of the guesswork involved in shopping. Indeed, concerns about the quality of fit rank first in the list when it comes to consumer reluctance to purchase clothes on-line. It is not surprising that customers who actually try on clothes in fitting rooms have a conversion rate of $67 \%$, compared to just $10 \%$ for those that do not [42]. In addition, return rates at a level of $15-50 \%$, depending on the type of item, cause significant financial loss for online retailers. It is interesting to note that approximately $60 \%$ of these returns claim improper fit as the reason for the return.

By assuring prospective customers of a "perfect fit", VTRs remove an important obstacle for online shopping and become perfect vehicles for increased sales and decreased return rates. Modern VFRs combine AR technologies with depth and color data to provide robust body recognition functionality and successfully address the fit and suit aspects of shopping. The article discusses successful examples of VFRs as well as the enabling technologies behind some of these shopping and advertising tools, many of which employ full-body scans to offer only pieces of clothing with a satisfactory fit and at the same time facilitate the styling and matching aspects of shopping.

These platforms are not only powerful decision tools for on-line shopping but also contribute to the fun factor for in-store shopping. In addition, the incorporation of social media in VTRs allows shoppers to obtain quick advice and feedback (e.g., Tweeter, Facebook) for a more pleasing and interactive shopping experience. Smartphone applications can also alert nearby stores to wishlisted items so that local shopping and deal finding can be usefully combined. 
The International Journal of Multimedia \& Its Applications (IJMA) Vol.4, No.4, August 2012

\section{ACKNOWLEDGEMENTS}

Work in this paper has been partially funded by the European Union and the Hellenic General Secretariat for Research and Technology (GSRT) under the "COOPERATION 2009 - 09SYN-72956" Framework.

\section{REFERENCES}

[1] Dimitris Protopsaltou, Christiane Luible, Marlene Arevalo, Nadia Magnenat-Thalmann (2002) "A body and garment creation method for an internet-based virtual fitting room", in Proc. Computer Graphics International 2002 (CGI '02), Springer, pp105-122.

[2] Frederic Cordier, Hyewon Seo, and NadiaMagnenat-Thalmann (2003) "Made-to-Measure Technologies for an Online Clothing Store", IEEE Computer Graphics and Applications, Vol. 23, No. 1, pp38-48.

[3] Yves Chiricota (2003) "Three-dimensional garment modelling using attribute mapping”, International Journal of Clothing Science and Technology, Vol. 15, No. 5, pp346-358.

[4] In Hwan Sul \& Tae Jin Kang (2005) "Interactive garment pattern design using virtual scissoring method", International Journal of Clothing Science and Technology, Vol. 18, No. 1, pp31-42.

[5] Nico Zink \& Alexandre Hardy (2007) "Cloth simulation and collision detection using geometry images", in Proc. of the 5th international conference on Computer graphics, virtual reality, visualisation and interaction in Africa, AFRIGRAPH '07, pp187-195

[6] Phoebe R. Apeagyei \& Rose Otieno (2007) "Usability of pattern customizing technology in the achievement and testing of fit for mass customisation", International Journal of Clothing Science and Technology, Vol. 11, No. 3, pp 349-365.

[7] Shang Liu, JiaChuan Sheng, Li He \& Fan Chen (2008) "Simulation of 3D Garment Based on Six Pieces of Bezier Curved Surfaces", in Proc. 2008 International Conference on Computer Science and Software Engineering, pp1082 -1085.

[8] Umberto Cugini, Monica Bordegoni \& Rossella Mana (2008) "The role of virtual prototyping and simulation in the fashion sector", International Journal on Interactive Design and Manufacturing, Vol. 2, No. 1, pp 33-38.

[9] Ching-I Cheng, Damon Shing-Min Liu, Chun-Hung Tsai \& Li-Ting Chen (2009) "A 3D Virtual Show Room for Online Apparel Retail Shop", in Proc. 2009 Annual Summit and Conference AsiaPacific Signal and Information Processing Association, APSIPA ASC 2009, pp193-199.

[10] Bo Begole, Takashi Matsumoto, Wei Zhang, Nicholas Yee, Juan Liu \& Maurice Chu (2009) "A 3D Virtual Show Room for Online Apparel Retail Shop”, in Proc. 13th International Conference on Human-Computer Interaction. Part IV: Interacting in Various Application Domains, pp448-457.

[11] Shi Min, Mao Tianlu \& Wang Zhaoqi (2010) “3D Interactive Clothing Animation”, in Proc. 2010 International Conference on Computer Application and System Modeling (ICCASM 2010), pp449455.

[12] Cody Robson, Ron Maharik, Alla Sheffer \& Nathan Carr (2011) "Context-aware garment modeling from sketches", Computers \& Graphics, Vol. 35, No. 3, pp604-613.

[13] Jiachuan Sheng \& Zheng Zhao (2011) "Fast Garment Rendering Based on Bezier Curved Surfaces", Journal of Information \& Computational Science, Vol. 8, No. 13, pp2501-2508.

[14] Stefan Hauswiesner, Matthias Straka \& Gerhard Reitmayr (2011) "Free viewpoint virtual try-on with commodity depth cameras", in Proc. of the 10th International Conference on Virtual Reality Continuum and Its Applications in Industry (VRCAI '11), pp23-30.

[15] Nobuyuki Umetani, Danny M. Kaufman, Takeo Igarashi \& Eitan Grinspun (2011) "Sensitive couture for interactive garment modeling and editing”, ACM Transactions on Graphics, Vol. 30, No. 4, pp90:1-90:11.

[16] Francisco Pereira, Catarina Silva \& Mário Alves (2011) "Virtual Fitting Room: Augmented Reality Techniques for e-Commerce", in Proc. of Conference on ENTERprise Information Systems (CENTERIS 2011), pp62-71.

[17] Differences between the kinect for windows sensor and the xbox sensor. [Online]. Available: http://www.microsoft.com/en-us/kinectforwindows/news/faq.aspx

[18] OpenNI organization. [Online]. Available: http://www.openni.org/

[19] Prime Sense Natural Interaction. [Online]. Available: http://www.primesense.com

[20] Willow Garage. [Online]. Available: http://www.willowgarage.com

[21] Side Kick. [Online]. Available: http://www.sidekick.co.il 
The International Journal of Multimedia \& Its Applications (IJMA) Vol.4, No.4, August 2012

[22] Asus Web Site. [Online]. Available: http://www.asus.com

[23] AppSide. [Online]. Available: http://www.app-side.com

[24] OpenNI arena. [Online]. Available: http://arena.openni.org

[25] R. A. Newcombe, S. Izadi, O. Hilliges, D. Molyneaux, D. Kim, A. J. Davison, P. Kohli, J. Shotton, S. Hodges, and A. Fitzgibbon, "Kinectfusion: Real-time dense surface mapping and tracking," in Proc. 10th IEEE International Symposium on Mixed and Augmented Reality (ISMAR'11), Basel, Switzerland, 2011, pp. 127-136.

[26] Kinect for Windows SKD. [Online]. Available: http://kinectforwindows.org

[27] Kinect SKD 1.5 to be released. [Online]. Available: http://blogs.msdn.com/b/kinectforwindows/archive/2012/03/26/what-s-ahead-a-sneak-peek.aspx

[28] Microsoft Kinect SKD vs PrimeSense OpenNI. [Online]. Available: http://www.brekel.com/?page $\mathrm{id}=671$

[29] R. B. Rusu and S. Cousins, "3D is here: Point Cloud Library (PCL)," in Proc. IEEE International Conference on Robotics and Automation (ICRA'11), Shanghai, China, 2011.

[30] Point Cloud Library (PCL). [Online]. Available: http://pointclouds.org/

[31] Kinect fusion versus PCL. [Online]. Available: http://pointclouds.org/ news/kinectfusion-opensource.html

[32] NVIDIA CUDA. [Online]. Available: http://www.nvidia.com/object/cuda home new.html

[33] Bodymetrics. [Online]. Available: http://www.bodymetrics.com

[34] Bodymetrics launches new body scanner using primesense technology. [Online]. Available: http://www.bodymetrics.com/assets/BodymetricsLaunch.pdf

[35] Bodymetrics turns the tv into a fitting room with 3d bodymapping. [Online]. Available: http://www.bodymetrics.com/assets/BodymetricsRazorfishCESFINALJan1012.pdf

[36] Fitting Reality. [Online]. Available: http://fittingreality.com

[37] Imagine That technologies. [Online]. Available: http://www.imaginethattechnologies.com/

[38] Total Immersion. [Online]. Available: http://www.t-immersion.com/

[39] Styku smart fitting room. [Online]. Available: http://www.styku.com/

[40] Tukatech apparel technology. [Online]. Available: http://www.tukatech.com/

[41] Deepinder Singh, Manish Bansal \& Namandeep Kaur, (2012) "Internet Retailing - New Era of Marketing”, International Journal of Marketing and Technology, Vol. 2, No. 3, pp 154-169.

[42] New york's fashion world discusses technology disruption. [Online]. Available: http://www.forbes.com/sites/sap/2012/05/04/fashion-disruption/

\section{Authors}

Ioannis Pachoulakis received a B.Sc. in Physics (1988) at the University of Crete, Greece, and a Ph.D. in Astrophysics (1996) and an M.Sc. in Engineering (1998), both from the University of Pennsylvania in the U.S.A. Since 2001, he serves as an Assistant Professor at the Department of Applied Informatics and Multimedia at TEI of Crete with mainstream interests in realistic multimedia applications, virtual reality and multimedia applications for science.

Kostas Kapetanakis is a graduate of the Department of Applied Informatics and Multimedia (2011) at TEI of Crete, working towards his Ph.D. degree in mobile and multimedia applications. 